\title{
COMMUNICATING MULTILEVEL EVACUATION CONTEXT USING SITUATED AUGMENTED REALITY
}

\author{
I. Lochhead ${ }^{1,}{ }^{*}$, N. Hedley ${ }^{1}$ \\ ${ }^{1}$ Department of Geography, Simon Fraser University, Burnaby, Canada - (iml, hedley)@ sfu.ca
}

Commission VI, WG VI/4

KEY WORDS: 3D Geovisualization; Mixed Reality; Emergency Management; Evacuation Plans; Cognitive Mapping

\begin{abstract}
:
Emergency preparedness is a fundamental component of a successful emergency management strategy. This includes a proactive communication strategy that informs all stakeholders of the emergency plan and helps translate that knowledge to real spaces. Communicating multilevel built environments can be difficult, as the architectural complexity creates problems for both visual and mental representations of networks in 3D space. Modern mobile technology offers emerging opportunities for emergency managers to develop and deploy $3 \mathrm{D}$ visualizations of multilevel spaces that preserve the topology of those spaces while adding the spatial context that allows the individual to better understand their position within it. In this paper, we present a collection of mixed reality (specifically augmented reality) geovisualizations that overcome the visual limitations associated with the traditional static 2D methods of communicating the evacuation plans of multilevel structures. We demonstrate how this technology can provide spatially contextualized 3D geovisualizations that promote spatial knowledge acquisition and support cognitive mapping. These geovisualizations are designed as a proactive emergency management tool to educate and prepare at risk populations prior to the occurrence of a hazardous event.
\end{abstract}

\section{INTRODUCTION}

The primary objective of any emergency management plan is to ensure the health and safety of the people. While there may be slight strategic variations between organizations, the foundation of those plans is typically formed around the core elements (mitigation, preparedness, response, and recovery) of the emergency management cycle. Regardless of what that plan is, or how it was developed, for the plan to be successful it must be effectively communicated to all those who are at risk. For indoor environments, that communication frequently involves strategically positioned notices of the emergency procedures, accompanied by a map that outlines those procedures in a simple visual depiction of that space. While there is a significant body of research that supports the development of emergency and evacuation plans, few have focused on the visual communication of that information. The traditional 2dimensional (2D) maps which are relied upon to communicate the evacuation plans of these complex 3-dimensional (3D) built spaces, provide planar perspectives that are limited in their ability to advance spatial knowledge acquisition, spatial awareness, and therefore, public safety within built environments.

While evacuation maps aim to inform the reader about their position in space, they often challenge the reader's comprehension of where, exactly they are. The "you-are-here" symbol or phrase that adorns countless evacuation maps can lead to significant confusion for the reader. In single-floor buildings a you-are-here map may be straightforward, as the mental connections between the visual depiction of the plan and the readers position within it, to the real-world space it represents, may be unchallenging. In multi-floor buildings, these maps can become extremely complex, often requiring the reader to connect their position above or below ground to evacuation routes on dissimilar levels, using abstract mental representations of those spaces. We argue that the traditional $2 \mathrm{D}$ visualizations that represent these complex, built spaces fail to adequately contextualize those 3D spaces, and that supplementary methods of visualization are needed to improve spatial cognizance and emergency preparedness in built spaces.

The objective of this paper is to present the research and development behind a series of mixed reality (MR) geovisualization systems for communicating emergency evacuation plans within multilevel spaces. We review current methods used to communicate evacuation information, focusing on their ability to represent complex spaces and encourage cognitive mapping. We then introduce MR as an emerging strategy for communicating geospatial information and highlight MR applications that improve one's ability to reason with, and better comprehend visual information, across multiple disciplines.

\section{MAIN BODY}

\subsection{Research Context}

As the world's population trends towards densified urban living and natural and manmade hazards become increasingly prevalent, there is greater potential for catastrophic events that impact large populations. Emergency plans strive to reduce the risk to these populations and evacuation plans attempt to guide people to safety; however, the plans themselves serve little purpose to the population facing those risks unless they understand them. In the absence of training, experience, or familiarity with a given space, maps are one method through which evacuation plans and other geospatial information can be communicated to the public (Dymon and Winter, 1993). However, transferring the information presented on those maps to the real-world can be difficult, and if poorly designed, these maps can leave the reader 'confused and disoriented' (Dent, 1972). Maps serve a vital role in communicating geospatial information to the public, yet pervasive modern mobile technology provides a platform and the opportunity for new perspectives, added dimensionality, and levels of spatial 
awareness that are difficult to achieve using traditional maps. This section presents research on the function of evacuation maps, the construction of cognitive maps, and the opportunity for MR geovisualizations to change the way we conceptualize complex multilevel spaces.

2.1.1 Evacuation Maps: Evacuation maps are designed to inform the public of the evacuation plan. They are one of three map styles (planning, evacuation, and crisis) characterised as a type of emergency map (Dymon, 1994). Evacuation maps for indoor environments are typically located within rooms and along main transportation corridors, highlighting the position of the reader and the pathway to the nearest exit in an effort to decrease egress time (Teknomo and Fernandez, 2012). Despite the reliance on these maps to communicate critical information, a review of the theoretical research behind evacuation map design reveals little more than an agreement that there is a lack of research on the topic. Most of this research focuses on outdoor environments at regional scales.

The published research on evacuation maps suggests that a successful map is one that is easily understood and adheres to basic cartographic principles (Chen et al., 2015; Dymon and Winter, 1993). In times of crises, these maps should provide a clear visualization that allows the reader to situate themselves and find safety without having to decipher lengthy text or confusing visuals (Dymon and Winter, 1993). However, in an analysis of the evacuation maps for areas surrounding 13 nuclear power stations in the United States, Dymon and Winter (1993) found that most failed to include basic map elements (e.g. a compass rose or legend) and downplayed risks by reducing the station's visibility on the map. These maps were not clear or easy to understand and were further burdened by policy guidelines that required lengthy prose for those unable to read maps. Their analysis concluded that the expected role served by evacuation maps was limited.

The evacuation map is therefore considered a resource for those that need assistance during an evacuation, and should not be used to educate the public about evacuation procedures. Yet, in the absence of the evacuation drills and exercises that have been identified as a critical component of emergency preparedness (Public Safety Canada 2010; FEMA 2013; University of Canterbury 2014, and others), there are limited resources or opportunities for educating the public about evacuation routes. Regardless of how clearly those routes are marked in the realworld, research indicates a reluctance to follow unknown pathways and a preference to retrace familiar ones, even if more direct routes to safety are available (Johnson, 2005). This suggests that the cognitive maps (mental representations of space) that a building's occupants are relying on to navigate these spaces are incomplete and that new methods of visualization, which promote higher levels of spatial cognizance, would be beneficial for improving emergency preparedness.

2.1.2 Cognitive Maps: Cognitive, or mental maps are a mental representation of space that one generates and stores in their mind. When faced with complex spatial challenges we call upon our memories of these spaces, the mental maps, to provide a birds-eye perspective (Taylor et al., 2008). The concept of cognitive mapping was first proposed in 1948 by American psychologist Edward Tolman, who theorized that rats, and by extension humans, develop mental representations of space that influence their behaviour within those environments. The idea of a cognitive map has since been widely adopted across the social sciences, resulting in contrasting definitions and a contested trend toward widespread use defining all thoughts and behaviours related to spatial environments (Kitchin, 1994).

The definition of a cognitive map, as well as the theories surrounding their development, varies across disciplines. While some research supports the concept of associative models that use the allocentric relationships between landmarks to codify space, others support positional models that define the egocentric relationship of one's self to the spatial landmarks of a given place (Taylor et al., 2008; Wang and Spelke, 2000). Others report that cognitive maps combine allocentric and egocentric representations according to the specifications of the task and the environment for which they are referenced (Burgess, 2006; Newman et al., 2007). Either way, the spatial knowledge necessary for cognitive map development can be obtained through direct interaction with real-world spaces, or through mediated interactions with visual representations of those spaces (Sharlin et al., 2009); irrespective of the source, a mental model is not the inevitable outcome (Taylor et al., 2008). Constructing mental representations of a given space is a cognitive challenge, especially when those spaces are complicated, multilevel, interconnected structures.

The scarcity of vacant space, combined with increasing human populations within and surrounding modern cities, necessitates vertical development characterised by increasingly large multilevel structures. These complex spaces can be frustrating, confusing, and difficult to navigate, particularly when attempting to travel between floors (Li and Giudice, 2012). Vidal et al. (2004) suggest that the cognitive maps for these multilevel spaces are composed of a series of 2D mental representations connected by junctions, and that the physical rotation and vertical displacement experienced when travelling in these spaces (e.g. stairwells) inhibits contiguous mental mapping. Without an external landmark (real or virtual) as a frame of reference, it is difficult for humans to mentally connect the multiple levels of built spaces (Li et al., 2016). These examples illustrate how the experiential knowledge gained navigating multilevel indoor spaces fails to yield the general frame of reference (survey knowledge) required to comprehend spatial relationships throughout complex spaces. As survey knowledge can be attained using visuals (e.g. maps or models), there is an opportunity to employ 3D models and MR interfaces to provide additional perspectives that support spatial knowledge development within these spaces (Huang et al., 2012).

2.1.3 Mixed Reality: MR technology has emerged as a hot topic in recent years, as new products from Google, Microsoft, Apple, and Magic Leap promise to merge real and virtual worlds in fascinating ways. The concepts of mixed and augmented reality are not new, and despite the seemingly interchangeable use of the terms, they are not one and the same. MR describes display technology that merges real and virtual environments. It was first introduced by Milgram and Kishino (1994) to describe an emerging collection of visual display systems, occupying the middle ground between entirely real and entirely virtual environments along their "virtuality continuum." They further subdivide MR as either augmented reality (AR) or augmented virtuality (AV) depending on the proportions of real and virtual content. The MR interfaces garnering recent attention add virtual objects to predominantly real-world environments and are therefore examples of AR. 
AR has many valuable applications beyond the gaming, social media, and marketing activities which have brought it into the mainstream. A review of AR applications by Billinghurst et al. (2015) highlights how the technology has been used by doctors to visualize the inside of the human body, by architects to see unfinished buildings, and by students to arrange virtual models of complex molecules in the classroom. These examples demonstrate how AR can be employed to help visualize that which cannot be seen, allowing the viewer to make sense of abstract phenomena. Similarly, Hedley (2008) identifies several geographic applications for the visualization of spatial phenomena, and more specifically, the use of mobile augmented reality (MAR) to display virtual geographic information in everyday spaces. These in-situ visualizations hold tremendous potential for improving our ability to understand and navigate complex multilevel spaces.

A key component of AR interfaces is their ability to register virtual objects to real environments, providing the illusion that both occupy the same space. This 'tracking' is critical for navigation purposes, as the virtual guidance provided by the MAR application must align with the real-world environment to which it applies. Outdoor MAR navigation systems use GPS signals to register virtual information to the user's position in that space (Dünser et al., 2012; Tsai et al., 2012); however, indoor MAR systems cannot rely on GPS signals, alternatively using Wi-fi signals to provide the necessary positional tracking (Torres-Sospedra et al., 2015). More recently, Google has developed a visual positioning service (VPS) for its Tango enabled devices that tracks the physical characteristics of realworld spaces and subsequently registers virtual information to them. Despite these advances, guidance systems may not be the best application of AR for emergency managers that are looking to increase a population's spatial awareness and overall safety in built environments.

While familiarity with space can produce the spatial knowledge used to generate cognitive maps, research has shown that GPS and automatic navigation systems do not improve spatial awareness (Huang et al., 2012; Speake and Axon, 2012), and that these systems can create 'passive operators' with a degraded ability to acquire spatial knowledge (Parush et al., 2007). If $A R$ is to be applied to emergency management with the purpose of improving spatial awareness, it must enhance the user's ability to comprehend the topology of complex multilevel spaces by providing allocentric representations of space instead of egocentric guidance through space.

2.1.4 Contextualizing Complex Spaces: The 2D you-arehere maps that are commonly used within multilevel structures provide static, disjointed, and often restricted representations of multilevel spaces. While these maps are important to public safety efforts, it could be argued that supplementary methods of visualizing these spaces are necessary to deliver the spatial context that is required to improve spatial awareness within complex built structures.

The preceding sections highlighted how evacuation maps are meant to inform evacuees in times of crises, providing a quick reference that helps them better understand the space and evacuate from it. People rely on cognitive maps when navigating space and the creation of these mental maps in complex multilevel structures can be challenging, often resulting in fragmented mental representations and a preference to evacuate via the familiar, rather than along the quickest or safest path. While evacuation exercises are important, it is impractical to suggest that these exercises can be conducted by all people, from all possible locations within a building. The following sections present the workflow behind a collection of AR geovisualizations that supplement the existing campus maps, signage, and evacuation plans at Simon Fraser University (SFU), Canada. The objective of these interfaces is to highlight how AR-based 3D evacuation visualizations, situated and specific to real spaces, may enhance spatial knowledge acquisition and cognitive mapping in complex multilevel buildings.

\subsection{Methodology}

Presented here are a collection of innovative AR prototypes for the visual communication of emergency evacuation information in a complex institutional space. The objective of this research is to demonstrate the application of MR interfaces within the realm of emergency management. This research builds upon the authors' previous emergency evacuation research exploring game-engine based evacuation simulations situated in real and virtual spaces. While that research addressed the influence of space on evacuation behaviour, this research explores a new communication strategy aimed at cultivating spatial awareness and influencing evacuation behaviour in multilevel space. The workflow introduced in the following subsections is outlined in Figure 1.

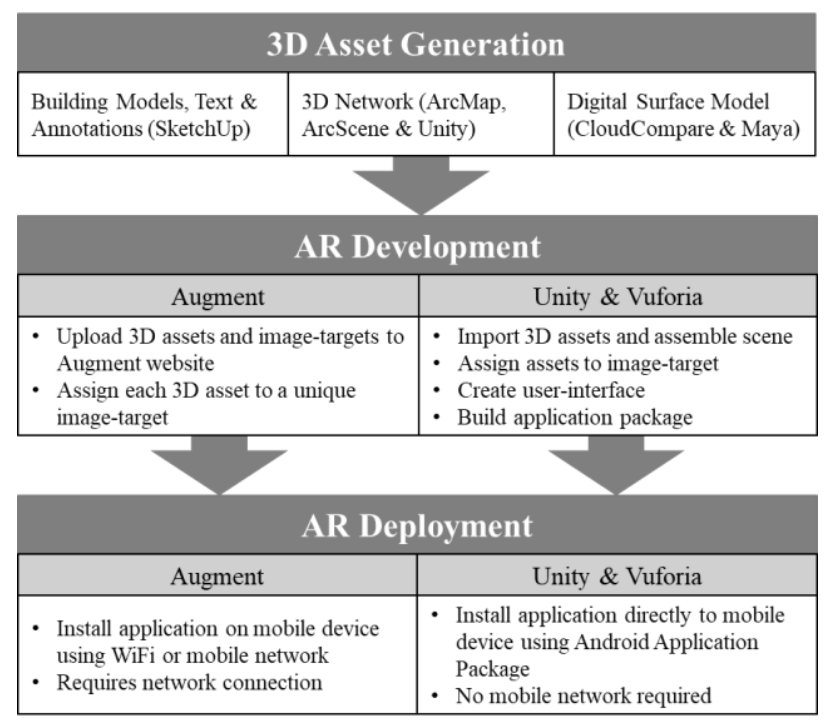

Figure 1. The workflow used to create the presented prototypes

2.2.1 3D Assets: The AR visualizations presented in this paper focus on the visual communication of 3D geospatial information, combining 3D GIScience with 3D modelling and game-engines.

The 3D model of SFU's Academic Quadrangle (AQ) that is used in these visualizations was developed using SketchUp design software. An architectural drawing (.dwg file) of each floor was imported into SketchUp and the 3D structure of the building was extruded according to the drawings and GPS measurements. SketchUp was also used to build the 3D assets highlighting the evacuation pathways in the first two examples. Both the 3D building and the evacuation pathways were exported as 3D Object files (.obj). The 3D model of SFU used in the campus wide evacuation map was provided by SFU Facility Services, but was modified within SketchUp to focus only on primary structures. The evacuation pathways and labels were added to this model by the authors. 
CloudCompare is an open source 3D point cloud and mesh processing program that was used to build the 3D surface model presented in example three. The LiDAR point cloud and highresolution image used to create this model were provided by SFU's Spatial Information Sciences department. The 3D model was clipped to its current extent, and textured, using Autodesk Maya.

The evacuation pathways presented in example three were derived from a 3D network analysis conducted in ArcScene. This network analysis calculated the five exits nearest to the location of the evacuation sign used as the AR image-target. The 3D shapefiles for those network segments were exported from ArcScene as 3D assets (Object files) using the Data Interoperability extension.

2.2.2 Mobile Deployment: The visualizations presented here were developed for Android powered mobile devices and could be reconfigured for Apple or Windows based hardware. The chosen operating system reflects the technology and developer permissions available to the authors. These prototypes were tested on two Samsung Galaxy mobile phones (S4 and S7). These mobile devices are typical of the compact, yet powerful mobile technology that has become a pervasive component of modern life.

2.2.3 Augmented Reality: The following prototypes provide examples of image-based AR (also known as tangible AR or marker-based AR), which uses computer vision software to identify pre-defined images, subsequently rendering virtual objects on the display system according to the position and orientation of those images in real space. One of the most common forms of AR image is a coded black-and-white design similar to a QR code (Cheng and Tsai, 2013); however, the presented prototypes use natural feature tracking and are designed to recognize visual patterns in the real-world based on photographs of those features (Hedley, 2017). This workflow would allow emergency managers to supplement existing infrastructure without having to modify it.

The first two examples were developed for Augment, a thirdparty AR application. The $3 \mathrm{D}$ assets were uploaded to a webbased database and can be downloaded directly to any mobile device containing the Augment software. Each virtual 3D object is associated with a specific real-world feature at SFU (room number, hallway marker, or campus map). The third example was developed with Unity, an open-source game engine, and the AR software development kit (SDK) offered by Vuforia. This prototype application was built on PC and deployed directly to the authors' Android smartphones. This software was programmed to recognize a specific evacuation plan sign posted at SFU; however, similar evacuation signs could be augmented with their own unique 3D datasets. Each of the presented AR prototypes are summarized in Table 1.

\begin{tabular}{|l|l|l|}
\hline Prototype Visualization & \multicolumn{1}{|c|}{ Application } & \multicolumn{1}{|c|}{ AR Platform } \\
\hline Campus Map & $\begin{array}{l}\text { Supplement 2D campus maps with } \\
\text { 3D building models and general } \\
\text { evacuation routes }\end{array}$ & Augment \\
\hline Evacuation Pathway & $\begin{array}{l}\text { Present 3D building models and } \\
\text { evacuation routes specific to the } \\
\text { user's location }\end{array}$ & Augment \\
\hline Evacuation Plan & $\begin{array}{l}\text { Transform static evacuation plans } \\
\text { into interactive learning tools }\end{array}$ & Unity and Vuforia \\
\hline
\end{tabular}

Table 1. Outline of presented AR prototypes

\subsection{AR Geovisualizations}

This research focused on developing a workflow and a series of prototype visualizations that facilitate the visual analysis of evacuation information, situated and specific to the location from which it is presented. The objective is to underscore how AR display technology can contextualize complex spaces by providing interactive $3 \mathrm{D}$ visualizations that maintain the interconnected nature of built space, both within and beyond the structural confines of those spaces. These visualizations exemplify a technique for presenting information about built spaces, preserving its dimensionality, and encouraging the cognitive connections between the abstract and the real.

2.3.1 Campus Maps: The first AR visualization was created to provide general evacuation information across a university campus. This includes simple 3D representations of the exterior of every major building on campus, labelled with distinct 3D block text, and illustrates the general evacuation pathway from each building to a safer location (Figure 2). The objective is to illustrate how traditional maps, fixed in their form and function, can become the foundation for interactive AR displays. With $\mathrm{AR}$, the theme of any map is no longer static, as each can be augmented with additional information and dimensionality. This visualization can be operated on any mobile device containing the Augment application.

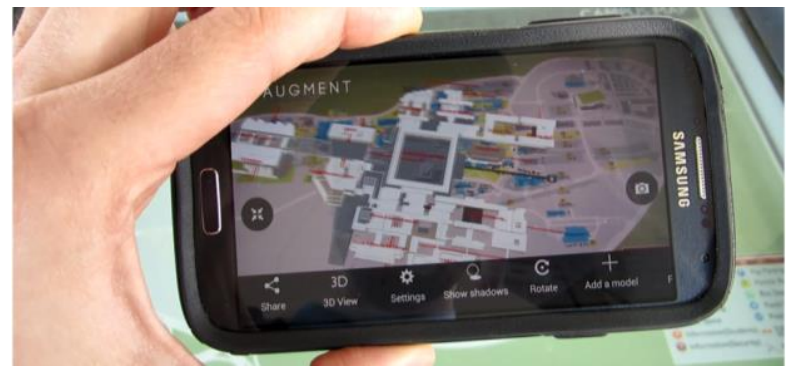

Figure 2. Augmented campus maps

In this example, the $3 \mathrm{D}$ data and an image of the campus map were uploaded to the Augment database, where the image was subsequently registered as the image-target that is used by the software to position and display the 3D objects. When those distinct visual patterns are detected, the 3D data is presented on the device's display. The user can then manipulate the position and orientation of that data directly on their device, or they can explore that data by adjusting the position of their device relative to the image-target (campus map).

This visualization was designed to educate people about the general evacuation procedures at SFU. To the authors' knowledge, there are no maps, or other visual tools, that outline campus wide evacuation plans. In complex built spaces where multiple buildings are interconnected, and one building's exit may place you on the roof of another, it is important that people understand the topology of space and the role it plays in safe and efficient evacuation pathing. AR provides an effective method of communication that harnesses the power of modern technology to extend the capacity of current emergency infrastructure without altering the complexity or quantity of existing emergency signage. 
2.3.2 Evacuation Pathways: The second AR visualization illustrates how AR can be used to provide situated evacuation information. The two visualizations in this example contain a 3D model of the AQ and the evacuation pathway from the viewer's location to a designated muster site (Figure 3 ). The objective here is to illustrate how AR can provide location specific geospatial information that encourages the cognitive connection between the presented information and the viewer's location within that space. With these visualizations the viewer is granted additional context in the form of a 3D representation of the entire building (not simply the floor they are on), an evacuation pathway out of that building (not the nearest stairwell or junction), and a suggested path to a muster site (not an assumption that outside equals safety).

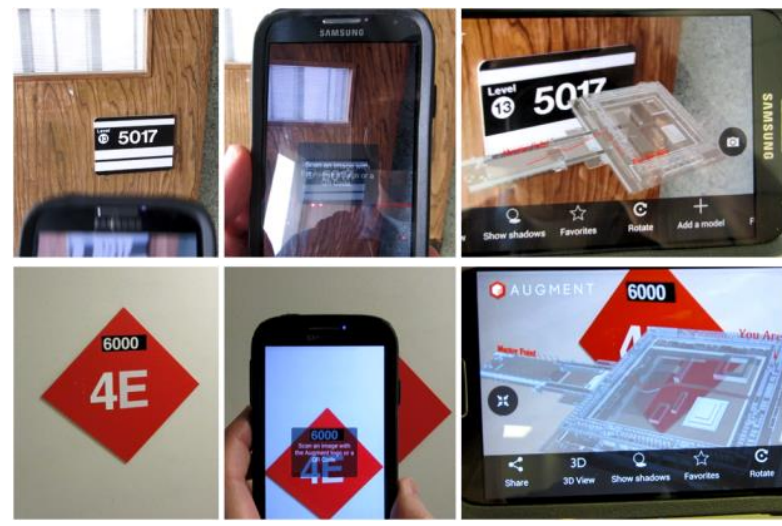

Figure 3. Augmenting the existing building signage

These visualizations operate using the same Augment application introduced in example one and follow a similar workflow. However, instead of using a campus map for AR registration these examples were designed to illustrate how the visual characteristics of inconspicuous objects within a building could become the stage for situated 3D visualizations. The room number displayed outside of a classroom and a sign identifying the floor number and location of a stairwell were used as the prerequisite visual features. Distinct visual targets like these are common across the SFU campus and within multilevel structures, providing an opportune platform for the delivery of situated geospatial information.

These visualizations were inspired by an apparent lack of evacuation drills at SFU. In the absence of evacuation exercises, people on campus are left to rely on their cognitive maps, or the cognitive maps of others, should an evacuation be necessary. Given the complex architectural characteristics of SFU, those cognitive maps may include disjointed and incomplete representations of space, representations incumbered by the existing 2D maps that provide a snapshot of these multilevel, interconnected spaces. The presented AR visualizations offer supplementary $3 \mathrm{D}$ perspectives that foster contiguous mental representations, allowing the viewer to make sense of the space they are within. These visualizations are not meant to replace existing evacuation maps or to be relied upon in times of crisis, but could be used as a tool for ongoing development of spatial awareness within complex built spaces.

2.3.3 Evacuation Plans: Evacuation plans are designed to inform people about the evacuation procedures for a given space. While there are few scientific research papers dictating or evaluating the design and content of evacuation plans, these plans are an essential component of emergency preparedness that can be found in most public spaces. The evacuation plan posted within SFU's AQ provides a written description of the emergency procedures and a simple 2D map of the evacuation plan that is specific to the location where that sign is posted (Figure 4). The map specifies the viewer's location (you-arehere) as well as the location of exits, the assembly area, and emergency equipment. The plan also suggests an evacuation route; however, the evacuation plan fails to provide the viewer with sufficient spatial context, demanding they connect their position to an outdated map absent of critical infrastructure updates such as the building that is now located on the suggested evacuation route. The third example of AR visualizations is a prototype interface for communicating evacuation plans.

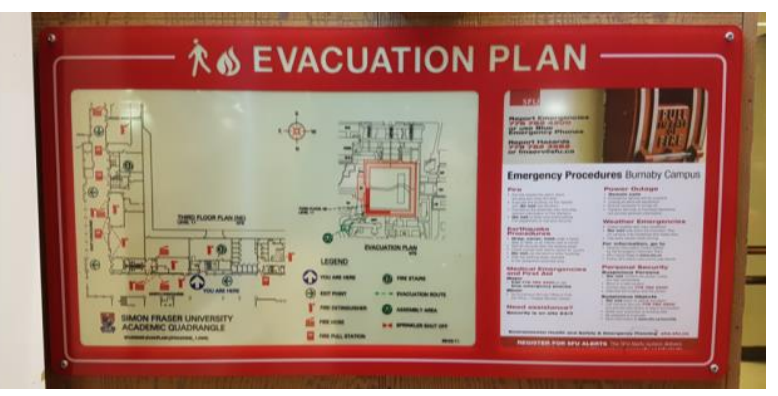

Figure 4. An example of the existing evacuation plan signage

The AR interface presented here is a mobile application that augments an evacuation plan with additional 3D geospatial data. It was developed using Unity along with an AR SDK from Vuforia, and was installed on the authors smartphone (Galaxy S7) using the Android and Java development kits. A photograph of the evacuation plan was converted into an AR image-target using the Vuforia Developers Portal. AR content is displayed on the mobile device when the user directs the device camera at the evacuation plan. In the following subsections, we discuss the different options for displaying AR content within this prototype application.

The first visualization provides a top-down perspective of a 3D model covering the same footprint, and contains the same information, as the 2D evacuation map on the posted evacuation plan (Figure 5). The 3D model aligns with the 2D evacuation map when it is displayed on the user's screen. The user can rotate that model and adjust its scale using the sliders on the graphical user interface (GUI). The user can also manipulate their perspective by adjusting the position of the mobile device relative to the evacuation plan. The objective of this visualization is to provide a visual depiction of the space that is less abstract. The added dimensionality and aerial imagery provide visual context that would better match the mental representation gained by experiencing that space, and different visual perspectives may help trigger cognitive connections between data and place. 


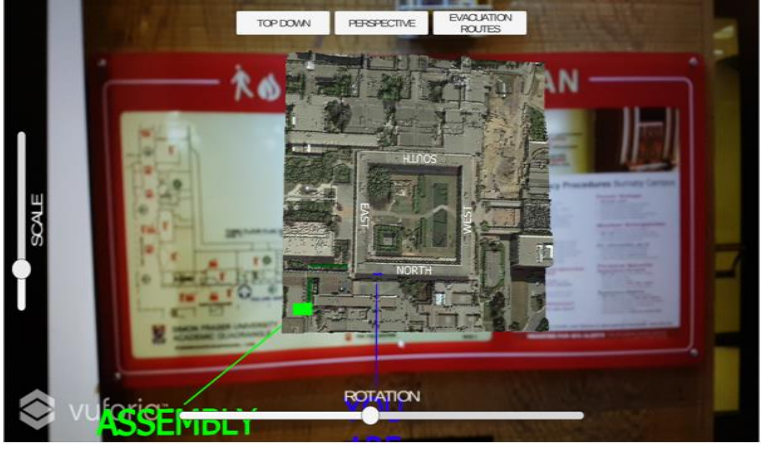

Figure 5. AR evacuation plan visualization (nadir)

The second visualization in this example utilizes the same 3D model as the first, only this time the spatial orientation of the model is aligned with the real-world (Figure 6). The user can manipulate the scale and rotation of the model with the GUI; however, orienting the 3D model with the real-world removes the need for mental rotation, an unnecessary cognitive load that can cause users to misinterpret the data (Lonergan and Hedley, 2015).

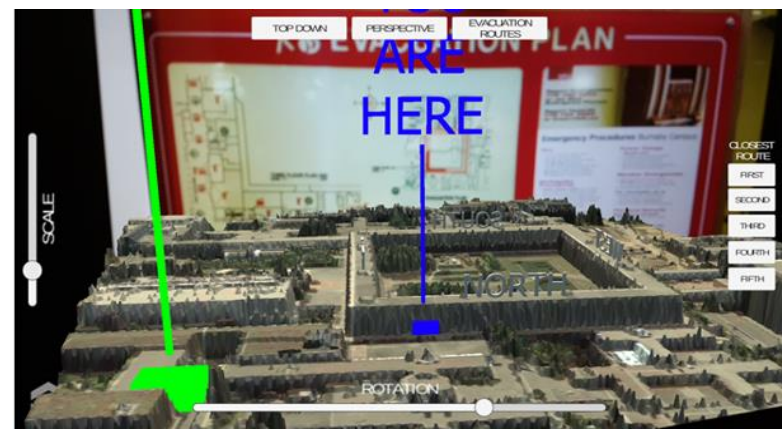

Figure 6. AR evacuation plan visualization (off-nadir)

An added GUI feature of this interface allows the viewer to explore the location of the five emergency exits closest to the viewer's position, as defined a by a 3D GIS network analysis (Figure 7). While the 2D map on the evacuation plan does indicate the location of exits, it does not provide the spatial context that allows the viewer to understand what lies outside those exits. The objective of this visualization is to provide a platform that allows people to better understand their position within the AQ, relate that position to the physical features on the outside, and make informed decisions should they need to evacuate the building and find safety.

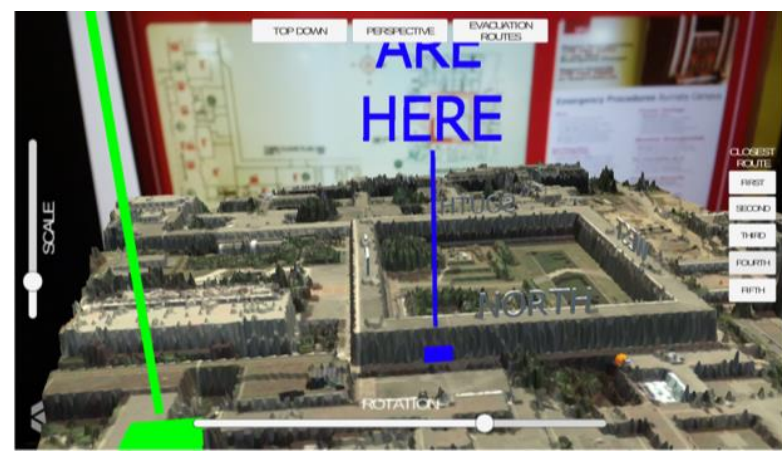

Figure 7. Spatially contextualized emergency exit locations

The final visualizations in this example provides a $3 \mathrm{D}$ representation of the internal features of the AQ. The model is rendered as a transparent mesh, allowing the viewer to observe the evacuation pathways leading from their position within the building to the five exits identified by the 3D GIS network analysis (Figure 8). The interface maintains the same GUI controls that allows the user to adjust the scale and spatial orientation of the model, and selectively display each of the five evacuation pathways.

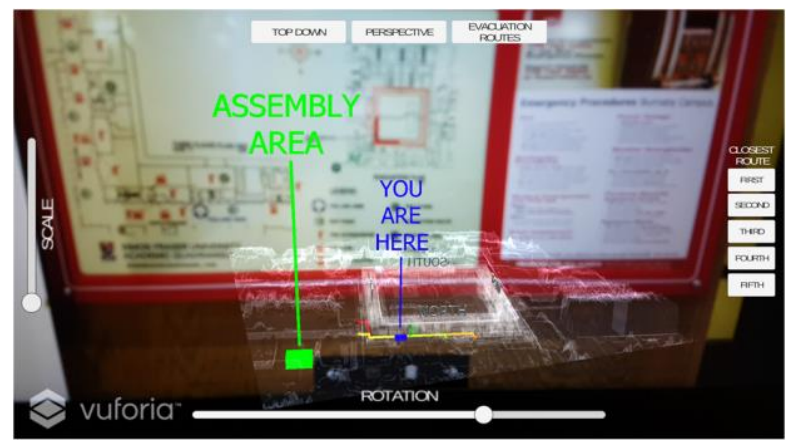

Figure 8 . Spatially contextualized evacuation pathways

The objective of this visualization is to add context to the evacuation pathway. Without context it is extremely difficult to know whether the exit by the stairwell identified on the 2D map is on the same level, above, or below the viewer. The transparent nature of the 3D model in this example allows the user to clearly identify that the closest exit can be accessed on the floor above via the nearby stairwell.

A video demonstrating each of the abovementioned prototypes can be found at https://youtu.be/AZv5zAI95LA.

\subsection{Discussion}

The examples presented in this paper demonstrate how AR may be used to facilitate spatial knowledge acquisition in complex spaces. We presented AR as a tool for communicating and learning abstract spatial information; not to be used in response to an emergency, but as a communication strategy for increasing emergency preparedness and public safety. In this paper, we used emergency evacuations as a framework to illustrate how AR technology can be applied to multidimensional network problems; however, AR holds great potential as a tool for communicating an array of geospatial information. The following sections provide a discussion on the affordances offered by AR, the limitations that were observed during the development and testing of these evacuation visualizations, and concludes with a recommendation for future research.

2.4.1 The Affordances of AR: AR is a display technology. It is a way to view computer-generated objects. The value of AR lies not in what it allows us to see, but how it allows us to see it. Azuma (1997) noted that AR enables what Fred Brooks dubbed intelligence amplification: using a computer to make things easier. The process of creating a mental model of a multilevel space, and then connecting abstract information to that space, is a cognitive challenge. As presented in this paper, AR offers a tool which simplifies that connection by providing visuals that maintain the dimensionality and continuity of multilevel space and the abstract information (evacuation plans) that relate to it.

Nevertheless, it is not the visualization itself, but the way that one interacts with that visualization that makes AR a powerful learning tool. Shelton and Hedley (2004) suggest that it is the visual, spatial, and sensorimotor feedback provided by interacting with the AR interface that drives knowledge acquisition. The ability to explore and physically interact with 
abstract or complex phenomena is therefore critical to improving our understanding of them. This is supported by research on the use of $\mathrm{AR}$ to teach anatomy, where $\mathrm{AR}$ interfaces were found to improve the overall understanding of complex human systems while decreasing the cognitive load on the students learning them (Kujukk et al., 2016). Similar sensemaking AR interfaces would be beneficial for those attempting to comprehend the complex networks within multilevel structures.

2.4.2 Limitations: AR has proven to be a valuable tool for the visual communication of complex, and often abstract, phenomena. The quality of those visualizations is defined not by their visual fidelity, but by how clearly, they communicate information and how well they support knowledge acquisition. During the development of these visualizations we identified two key limitations: packaged AR software restricts implementation of nuanced geovisualization design and interface functionality and large or immovable image-targets inhibit tangible interaction.

The 3D visualizations presented in this paper were deployed to either a third-party or custom-built mobile application. While there are several AR applications on the market that can display 3D buildings and evacuation information, we highlight the limited functionality of those platforms as learning tools. Many are designed as marketing tools, or as a novel way to supplement a consumer product with virtual information. The software can handle animations and allows the user to manipulate the size and position of the content, but each image is associated with one data set and it lacks the interface controls that facilitate higher levels of interaction and analysis. The final set of visualizations, developed using Unity, offer a more GISlike experience that allows the user to activate different layers and switch between data products (all of which were registered to the same image-target).

Despite this onscreen interaction, the position of the imagetarget used in each of these examples was fixed in space. Tangible AR, or AR that allows the user to pick-up the imagetarget and interact with the virtual content (Hedley, 2003), increases the level of interaction and promotes investigation and sensemaking. However, increased interaction is a double-edged sword, as the ability to manipulate the image-target reduces the developer's ability to align the virtual content with the realworld, which then degrades the connection between abstract information and the real-world.

2.4.3 Application to Emergency Management: This research demonstrates that $\mathrm{AR}$ visualizations could be used to supplement the existing emergency communication strategies in built spaces. Whether it should, remains to be answered. Further research is required that tests the capacity of these visualizations to communicate geospatial information in a way that improves the viewers spatial and emergency cognizance. The next phase of this project will be to conduct those empirical tests.

Developing AR applications for emergency management and public safety programs does not guarantee public adoption. Much like the maligned QR code, AR technology requires special applications and additional effort from the user. We are not yet at a point (although it may seem like it) where the realworld is intertwined with a virtual one. If, or when, AR devices that continuously occupy our field of view become integrated with our daily life, AR interfaces such as these will become common place.

\section{CONCLUSION}

This paper presented the research and development behind a series of prototype AR geovisualizations for the communication of emergency evacuation information situated and specific to real-world spaces. This work serves to highlight the ability to represent complex multilevel spaces in their inherently $3 \mathrm{D}$ form using AR technology. We discussed the importance of emergency preparedness and the role of evacuation maps, the challenges associated with cognitive mapping in complex built environments, and the ability to display, interact with, and explore abstract information using AR. We then introduced a series of AR geovisualizations that were designed to enhance spatial perception and situational awareness of multilevel spaces through proprioceptive affordances of situated AR evacuation displays, to act as a demonstration of how AR tools may support improved emergency preparedness communication

$\mathrm{AR}$ is an emerging technology that has the potential to transform the way we interact with information about the built environment. The 2D maps that characterize these spaces and which currently form a foundation for our mental representations of them, could be used as the stepping stone towards interactive 3D representations that encourage greater levels of spatial awareness in multilevel space. We hope that others take this research as inspiration for future applications of AR in emergency management, not to blindly guide us through space, but to better develop our understanding of space, mitigate risk, and improve public safety.

\section{REFERENCES}

Azuma, R., 1997. A survey of augmented reality. Presence Teleoperators Virtual Environ. 6, 355-385. doi:10.1.1.30.4999

Billinghurst, M., Clark, A., Lee, G., 2015. A Survey of Augmented Reality, in: Foundations and Trends® in HumanComputer Interaction. pp. 73-272. doi:10.1561/1100000049

Burgess, N., 2006. Spatial memory: how egocentric and allocentric combine. Trends Cogn. Sci. 10, 551-557. doi:10.1016/j.tics.2006.10.005

Chen, Y.-H., Zick, S.E., Benjamin, A.R., 2015. A comprehensive cartographic approach to evacuation map creation for Hurricane Ike in Galveston County, Texas. Cartogr. $\begin{array}{llll}\text { Geogr. Inf. } & \text { Sci. } & \text { 406, } & \end{array}$ doi:10.1080/15230406.2015.1014426

Cheng, K.-H., Tsai, C.-C., 2013. Affordances of Augmented Reality in Science Learning: Suggestions for Future Research. J. Sci. Educ. Technol. 22, 449-462. doi:10.1007/s10956-0129405-9

Dent, B.D., 1972. Visual Organization and Thematic Map Communication. Ann. Assoc. Am. Geogr. 62, 79-93.

Dünser, A., Billinghurst, M., Wen, J., Lehtinen, V., Nurminen, A., 2012. Exploring the use of handheld AR for outdoor navigation. Comput. Graph. 36, 1084-1095. doi:10.1016/j.cag.2012.10.001 
Dymon, U.J., 1994. Mapping--The Missing Link in Reducing Risk under SARA III. Risk 5, 337.

Dymon, U.J., Winter, N.L., 1993. Evacuation Mapping: The Utility of Guidelines. Disasters 17, 12-24. doi:10.1111/j.14677717.1993.tb00484.x

Federal Emergency Management Agency, 2013. Guide for Developing High-Quality School Emergency Operation Plans, U.S. Department of Education, Office of Elementary and Secondary Education, Office of Safe and Healthy Students. Washington, DC. doi:10.1017/CBO9781107415324.004

Hedley, N., 2017. Augmented Reality. In Richardson D, Castree N, Goodchild M, Kobayashi A, Liu W, Marston R.(eds.) International Encyclopedia of Geography. 1: 1-13. Wiley and Sons/Association of American Geographers. doi:10.1201/9780849375477.ch213〈r10.1201/9780849375477. $\operatorname{ch} 213$

Hedley, N., 2008. Real-time Reification: How Mobile Augmented Reality May Change Our Relationship with Geographic Space, in: 2nd International Symposium on Geospatial Mixed Reality, 28-29 August, Laval University. Quebec City, Quebec.

Hedley, N., 2003. Empirical Evidence of Advanced Geographic Visualization Interface Use, in: Proceedings of the $21 \mathrm{st}$ International Cartographic Conference (ICC) "Cartographic Renaissance.” Durban, South Africa, pp. 10-16.

Huang, H., Schmidt, M., Gartner, G., 2012. Spatial Knowledge Acquisition with Mobile Maps, Augmented Reality and Voice in the Context of GPS-based Pedestrian Navigation: Results from a Field Test. Cartogr. Geogr. Inf. Sci. 39, 107-116. doi:10.1559/15230406392107

Johnson, C.W., 2005. Lessons from the evacuation of the world trade centre, 9/11 2001 for the development of computer-based simulations. Cogn. Technol. Work 7, 214-240. doi:10.1007/s10111-005-0009-5

Kitchin, R.M., 1994. Cognitive maps: What are they and why study them? J. Environ. Psychol. 14, 1-19. doi:10.1016/S02724944(05)80194-X

Kujukk, S., Kapakin, S., Goktas, Y., 2016. Learning anatomy via mobile augmented reality: Effects on achievement and cognitive load. Anat. Sci. Educ. 9, 411-421. doi:10.1002/ase.1603

Li, H., Corey, R.R., Giudice, U., Giudice, N.A., 2016. Assessment of Visualization Interfaces for Assisting the Development of Multi-level Cognitive Maps, in: Foundations of Augmented Cognition. Neuroergonomics and Operational Neuroscience. pp. 308-321. doi:10.1007/978-3-642-02812-0

Li, H., Giudice, N. a., 2012. Using mobile 3D visualization techniques to facilitate multi-level cognitive map development of complex indoor spaces, in: CEUR Workshop Proceedings, Spatial Knowledge Acquisition with Limited Information Displays. Kloster Seeon, Germany, pp. 31-36.

Lonergan, C., Hedley, N., 2015. Navigating the future of tsunami risk communication: using dimensionality, interactivity and situatedness to interface with society. Nat. Hazards 78, 179-201. doi:10.1007/s11069-015-1709-7

Milgram, P., Kishino, F., 1994. A Taxonomy of Mixed Reality Visual Displays. IEICE (Institute Electron. Inf. Commun. Eng. Trans. Inf. Syst.

Newman, E.L., Caplan, J.B., Kirschen, M.P., Korolev, I.O., Sekuler, R., Kahana, M.J., 2007. Learning your way around town: How virtual taxicab drivers learn to use both layout and landmark information. Cognition 104, 231-253. doi:10.1016/j.cognition.2006.05.013

Parush, A., Ahuvia, S., Erev, I., 2007. Degradation in Spatial Knowledge Acquisition When Using Automatic Navigation Systems. Spat. Inf. Theory 238-254. doi:10.1007/978-3-54074788-8_15

Public Safety Canada, 2010. Emergency Management Planning Guide 2010-2011.

Sharlin, E., Watson, B., Sutphen, S., Liu, L., Lederer, R., Frazer, J., 2009. A tangible user interface for assessing cognitive mapping ability. Int. J. Hum. Comput. Stud. 67, 269278. doi:10.1016/j.ijhcs.2008.09.014

Shelton, B.E., Hedley, N.R., 2004. Exploring a cognitive basis for learning spatial relationships with augmented reality. Technol. Instr. Cogn. Learn. 1, 323-357.

Speake, J., Axon, S., 2012. "I Never Use 'Maps' Anymore": Engaging with Sat Nav Technologies and the Implications for Cartographic Literacy and Spatial Awareness. Cartogr. J. 49, 326-336. doi:10.1179/1743277412Y.0000000021

Taylor, H.A., Brunyé, T.T., Taylor, S.T., 2008. Spatial Mental Representation: Implications for Navigation System Design, in: Reviews of Human Factors and Ergonomics. pp. 1-40. doi:10.1518/155723408X342835

Teknomo, K., Fernandez, P., 2012. Simulating optimum egress time. Saf. Sci. 50, 1228-1236. doi:10.1016/j.ssci.2011.12.025

Torres-Sospedra, J., Avariento, J., Rambla, D., Montoliu, R., Casteleyn, S., Benedito-Bordonau, M., Gould, M., Huerta, J., 2015. Enhancing integrated indoor/outdoor mobility in a smart campus. Int. J. Geogr. Inf. Sci. 8816, 1-14. doi:10.1080/13658816.2015.1049541

Tsai, M.-K., Lee, Y.-C., Lu, C.-H., Chen, M.-H., Chou, T.-Y., Yau, N.-J., 2012. Integrating geographical information and augmented reality techniques for mobile escape guidelines on nuclear accident sites. J. Environ. Radioact. 109, 36-44. doi:10.1016/j.jenvrad.2011.12.025

University of Canterbury, 2014. Emergency Response Plan. Christchurch, NZ.

Vidal, M., Amorim, M.A., Berthoz, A., 2004. Navigating in a virtual three-dimensional maze: How do egocentric and allocentric reference frames interact? Cogn. Brain Res. 19, 244 258. doi:10.1016/j.cogbrainres.2003.12.006

Wang, R.F., Spelke, E.S., 2000. Updating egocentric representations in human navigation. Cognition 77, 215-250. doi:10.1016/S0010-0277(00)00105-0. 